\title{
Intragastric Balloon Treatment for Obesity: Prospective Single-Center Study Findings
}

\author{
Yasin Peker $^{\mathrm{a}} \quad$ Evren Durak $^{\mathrm{b}} \quad$ Uğur Özgürbüz \\ a Third General Surgery Clinic, \\ ${ }^{\mathrm{b}}$ First General Surgery Clinic, \\ ' Department of Anesthesiology, Second Anesthesiology and Reanimation Clinic, Atatürk Training and Research Hospital, İzmir, Turkey
}

\section{Key Words}

Morbid obesity · Obesity · Intragastric balloon . Weight loss

\section{Summary}

Background: The intragastric balloon (IGB) procedure is an obesity treatment. Methods: A BioEnteric IGB was used in 33 patients between February 2006 and February 2009. Results: Of the 31 patients, 19 were female $(61.3 \%)$. Mean age was $35.48 \pm 9.31$ years. Following intravenous sedation, the balloon was inserted and inflated under direct vision by using saline $(600 \mathrm{ml})$ and methylene blue $(10 \mathrm{ml})$ solution. Average weight and mean BMI scores were as follows: $119.34 \pm 22.64$ (range 80-170) $\mathrm{kg}$ and $41.84 \pm 8.28$ (range 30-63.2) kg/m². Mean weight and BMI were measured as $104.31 \pm 21.33$ (range 64-151) kg and $36.43 \pm 7.36$ (range 26-52) kg/m² 6 months after the index procedure. Percent of excess weight loss (\%EWL) and percent of excess body mass index loss (\%EBMIL) were as follows: $29.16 \pm 15.99 \%$ (range $0.00-56.91 \%)$ and $35.45 \pm 19.46 \%(0-75.2 \%)$, respectively. All patients lost weight constantly for the 6-month period. Patients showed statistically significant weight and BMI losses for the first 3-month period but these decrements reached a plateau between the 4th and 6th month. Weight loss was not statistically significant during the second 3-month period. Few patients had mild complaints following balloon insertion; there was no balloon intolerance. Conclusions: IGB is safe and effective for short-term weight reduction in obese patients. Weight reduction during the second half of the treatment period needs closer follow-up.

\section{Introduction}

Intragastric balloon (IGB) treatment for obesity was first performed in 1982 by Ribben [1]. Since then, it has been used by several specialized centers, and its merits and disadvantages over other methods have been reported [2]. The method had to be abandoned in the late 1980s in the USA because of complications, but research continued in Europe resulting in a better understanding of its effects and the definition of suitable patients [2]. The potential benefit of IGB treatment is a sustained $5-10 \%$ weight loss in order to prevent obesity, and related co-morbidities and diseases [3]. Many years of research and design modifications have resulted in a growing interest from surgeons in recent years and increased use of IGB treatment [2]. However, many complications have been reported in relation to this procedure, and it is important to be familiar with managing these problems since they cannot be entirely avoided despite the best efforts [3-7]. In this report, we outline the results of 31 cases of IGB treatment for obesity.

\section{Material and Methods}

The BioEnteric Intragastric Balloon (BIB ${ }^{\circledR}$; Inamed, Allergan, Irvine, CA, USS) was used in 33 patients between February 2006 and February 2009. All procedures were performed by the same surgeon(Y.P.) in a private institution. Informed consent was obtained from all patients before the procedure. The IGB was removed in 2 patients because of family traditions, and those patients were excluded from the group. Of the remaining 31 patients, 19 (61\%) were female. Mean age \pm standard deviation (SD) was $35.48 \pm 9.31$ (range $18-52$ ) years.

\section{Balloon Insertion and Removal}

Following intravenous sedation with midazolam and propofol, an upper gastrointestinal endoscopy was performed (Fujinon 201, Tokyo, Japan) to exclude anomalies. The balloon was inserted by using specially designed

\begin{tabular}{ll}
\hline KARGER & $\oplus$ 2010 S. Karger GmbH, Freiburg \\
Fax +497614520714 & Accessible online at: \\
Information@Karger.de & www.karger.com/ofa \\
www.karger.com &
\end{tabular}


sheath while in a collapsed position. Then, the endoscope was re-inserted, and the balloon was inflated under direct vision with saline $(600 \mathrm{ml})$ and methylene blue $(10 \mathrm{ml})$ solution. Patients were allowed to stay in hospital overnight, and were given saline, proton pump inhibitor (PPI), and antiemetic and antispasmodic agents intravenously as suggested by Ohta et al. [8].

Follow-up was once a week for 1 month and then every 2 weeks for a 6-month period. During the follow-up visits, adaptation to the balloon, weight loss, diet changes, and complaints were evaluated. Dietician follow-up visits consisted of nutrition plan, nutritional education, and weight control assessment. $150 \mathrm{~min} /$ week mild exercise was planned in order to enhance physical activity.

Balloon removal was scheduled for 6 months after index placement. The patients were sedated, and by using a specially designed IGB removal instrument (Kobi Medizintechnik GmBH, Lehrte, Germany) and a conventional single-channel endoscope, the balloon was removed. There were no immediate complications following removal. IGB exclusion criteria are listed in table 1. Percent of excess weight loss (\%EWL) and percent of excess body mass index loss (\%EBMIL) were calculated according to the formula by Deitel [9].

\section{Statistical Analysis}

The statistical Package for Social Sciences for Windows 10.0 program was used for statistical analysis. Descriptive data was given as mean \pm SD. Student $t$ test and Mann Whitney U test (for non-Gaussian data) were

Table 1. IGB exclusion criteria
Age $\leq 18$ and $\geq 60$ years

$\mathrm{BMI}<30 \mathrm{~kg} / \mathrm{m}^{2}$

Progress via diet and other methods

Malignancy within last 5 years

Hormonal or genetic obesity

Pregnancy

Major psychiatric disease

Alcohol and drug abuse

Hiatal hernia $>5 \mathrm{~cm}$

Esophagitis (grade C-D)

Peptic ulcer, esophageal varicose changes

Abdominal surgery history

Anticoagulant or NSAID use

Table 2. Anthropometric findings and results (mean $\pm \mathrm{SD}$ )

\begin{tabular}{lccl}
\hline & Beginning & At the end of 6 months & $\mathrm{p}$ value \\
\hline Weight, $\mathrm{kg}$ & $119.34 \pm 22.64$ & $104.31 \pm 2.33$ & 0.000 \\
$\mathrm{BMI}, \mathrm{kg} / \mathrm{m}^{2}$ & $41.84 \pm 8.28$ & $36.43 \pm 7.36$ & 0.000 \\
\hline
\end{tabular}

used to compare the groups. Paired simple $t$ test was used to compare the data for each group individually. A p value less than 0.05 was considered significant.

\section{Results}

Mean age was $35.48 \pm 9.31$ (range 18-52) years. Average weight and mean BMI scores were as follows: $119.34 \pm 22.64$ (range $80-170) \mathrm{kg}$ and $41.84 \pm 8.28$ (range $30-63.2$ ) $\mathrm{kg} / \mathrm{m}^{2}$. Mean weight and BMI were measured as $104.31 \pm 21.33$ (range 64-151) $\mathrm{kg}$ and $36.43 \pm 7.36$ (range 26-52) $\mathrm{kg} / \mathrm{m}^{2}$ 6 months after the index procedure. The 6 -month reduction in weight and BMI scores were statistically significant $(\mathrm{p}=0.000)$ (table 2). \%EWL and \%EBMIL were as follows: $29.16 \pm$ $15.99 \%$ (range $0.00-56.91 \%$ ) and $35.45 \pm 19.46 \%(0-75.2 \%$ ), respectively. All patients lost weight constantly for the 6-month period. Patients showed statistically significant weight and BMI losses for the first 3-month period, but these decrements reached to a plateau between the 4th and 6th month. As seen in table 3 and figure 1, weight loss was not statistically significant during the second 3-month period. As outlined in table 4, weight loss and reduction in BMI were more effective in morbidly obese patients compare to the obese group (BMI $>40 \mathrm{~kg} / \mathrm{m}^{2}$ vs. $\left.30-39.9 \mathrm{~kg} / \mathrm{m}^{2}\right)$. \%EWL and $\%$ EBMIL rates were more effective in the obese group (table 4). Few patients had mild complaints following balloon insertion; there was no balloon intolerance. Complications are listed in table 5 .

\section{Discussion}

IGB treatment was first reported in 1982 [9]. Research was inspired by the discovery that people with gastric bezoars constantly intake limited amounts of food $[1,5]$. The IGB was initially designed to maintain weight loss in patients who do not respond to conservative therapy but are not yet candidates for surgical intervention $[6,10]$. The IGB system involves distension of the stomach, which subsequently induces a feeling of early satiety and leads to weight loss [9]. It has completed its evolution with the design of a variety of sizes, durable materials, and inflatability with fluid or air [2]. The liquid-inflated

Table 3. Monthly variations of anthropometric measurements (mean \pm SD)

\begin{tabular}{lllllll}
\hline & Day 0 to 1st month & 1st to 2nd month & 2nd to 3rd month & 3rd to 4th month & 4th to 5th month & 5th to 6th month \\
\hline Weight loss, $\mathrm{kg}$ & $\begin{array}{l}6.84 \pm 4.01 \\
\mathrm{p}=0.000\end{array}$ & $\begin{array}{l}3.17 \pm 3.03 \\
\mathrm{p}=0.000\end{array}$ & $\begin{array}{l}2.16 \pm 2.78 \\
\mathrm{p}=0.000\end{array}$ & $\begin{array}{l}0.92 \pm 2.80 \\
\mathrm{p}=0.079\end{array}$ & $\begin{array}{l}0.70 \pm 2.15 \\
\mathrm{p}=0.079\end{array}$ & $\mathrm{p}=0.031$ \\
BMI loss, $\mathrm{kg} / \mathrm{m}^{2}$ & $2.43 \pm 1.46$ & $1.12 \pm 1.11$ & $0.76 \pm 0.96$ & $0.30 \pm 1.01$ & $0.26 \pm 0.74$ & $0.54 \pm 1.18$ \\
& $\mathrm{p}=0.000$ & $\mathrm{p}=0.000$ & $\mathrm{p}=0.000$ & $\mathrm{p}=0.106$ & $\mathrm{p}=0.064$ & $\mathrm{p}=0.018$ \\
\%EBMIL & & $8.23 \pm 8.39$ & $4.53 \pm 5.41$ & $1.81 \pm 5.26$ & $0.97 \pm 5.10$ & $2.82 \pm 8.15$ \\
& & $\mathrm{p}: 0.000$ & $\mathrm{p}=0.000$ & $\mathrm{p}=0.065$ & $\mathrm{p}=0.298$ & $\mathrm{p}=0.064$ \\
\hline
\end{tabular}


Fig 1. Monthly variations of anthropometric measurements.

Table 4. Weight loss achieved by IGB in obese versus morbidly obese patients (mean $\pm \mathrm{SD}$ )

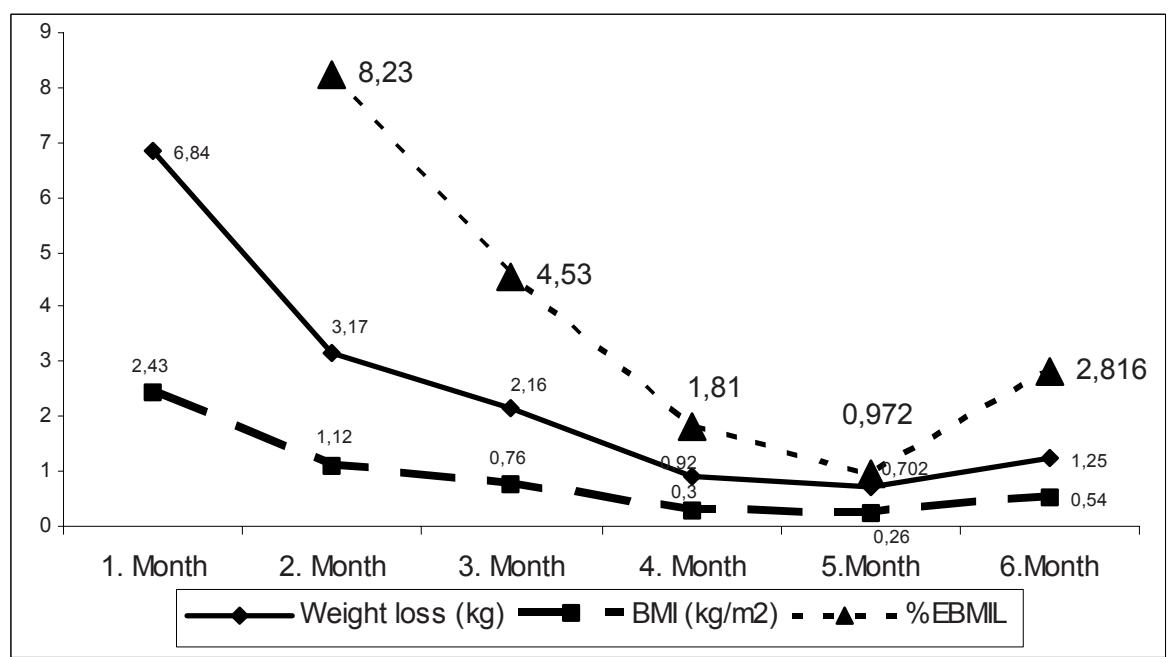

\begin{tabular}{lllll}
\hline & Weight loss, kg & BMI loss, $\mathrm{kg} / \mathrm{m}^{2}$ & \%EWL & \%EBMIL \\
\hline Obese $^{\mathrm{a}}(\mathrm{n}=19)$ & $12.8 \pm 6.86$ & $4.43 \pm 2.18$ & $32.12 \pm 15.08$ & $40.83 \pm 18.37$ \\
Morbidly obese $^{\mathrm{b}}(\mathrm{n}=12)$ & $18.55 \pm 12.66$ & $6.95 \pm 4.84$ & $24.44 \pm 16.89$ & $26.92 \pm 18.73$ \\
\hline${ }^{\mathrm{a}} \mathrm{BMI} 30-39.9 \mathrm{~kg} / \mathrm{m}^{2}$. & & & & \\
${ }^{\mathrm{b}} \mathrm{BMI}>40 \mathrm{~kg} / \mathrm{m}^{2}$. & & & & \\
\hline
\end{tabular}

type is most commonly used, and was also used in our study. It has many merits such as outpatient application, reversibility, re-applicability, lower rates of gastrointestinal and systemic complications, and decreased food consummation $[1,4$, $6,10,11]$. The IGB has been also used as preparation for major surgical procedures to minimize anesthesia risk [6], and it has been indicated that it can be safely applied in morbidly obese patients before bariatric procedures to decrease morbidity and mortality following definitive surgery [12, 13]. Left hepatic lobe volume decrease facilitates the laparoscopic procedures [3].

Over a period of 3 years, we have examined the results of 31 patients who underwent IGB treatment. We have demonstrated that IGB placement can be performed safely and effectively in a variety of obese patients since our patients' BMI score distribution ranges between 30 and $62 \mathrm{~kg} / \mathrm{m}^{2}$. Although clinical data concerning the benefits and complications of IGB remain scarce, our patients lost significant weight during the first 3 months after placement. The weight reduction rate was slowed between the 4th and 6th month but remained promising, especially for the morbidly obese patients. This phenomenon can be explained by an adaptation to the balloon that takes place over a period of 3 months. As a result, weight reduction during the second half of the treatment period needs closer follow-up. All balloons were removed 6 months after their placement, according to our protocol. According to a recent report by Göttig et al. [10], BMI changes or increases in \% EBMIL were not statistically significant in patients whose IGB therapy lasted longer than 200 days.
Table 5. Complications caused by the IGB

\begin{tabular}{llr}
\hline & $\mathrm{n}$ & $\%$ \\
\hline Nausea and vomiting over 1 week & 6 & 19.35 \\
$\begin{array}{l}\text { Abdominal pain and gastrointestinal system-related } \\
\quad \text { mild complaints }\end{array}$ & 6 & 19.35 \\
Constipation and/or diarrhea & 6 & 19.35 \\
Gastroesophageal reflux & 5 & 16.12 \\
Discharge and spontaneous ejection & 1 & 3.22 \\
\hline
\end{tabular}

${ }^{a}$ Dyspepsia, burning sensation at the back of the sternum, halitosis, flatulence.

In all our patients, the IGB was placed and positioned effortlessly and with minimal sedation. It was well tolerated, and only few patients suffered from mild gastrointestinal complaints. These were easily managed with PPI and antiemetic agents. There were no ulcers, mucosal erosions, or gastric perforations in our patients, and no mortality. In 1 patient, the IGB was spontaneously deployed via the feces and was replaced with a new one in an outpatient fashion. The latter is one of the features of IGB treatment since it can be re-applied to the same patient repeatedly with an acceptable complication rate $[4,6]$.

It has been clearly reported that this method is less efficient compared to definitive bariatric procedures [7]. On the other hand, its efficacy can be increased by adding strict diet regulations and behavioral treatment [7, 13-15]. Genco et al. [16] stated that sleeve gastrectomy or IGB treatment 12 
months prior to gastric bypass have similar efficiency rates. Our results indicate that IGB is safe and effective for shortterm weight reduction in obese patients. Weight reduction during the second half of the treatment period needs closer follow-up. The IGB can be easily placed by an experienced endoscopist or via minimally invasive surgery. Although the presented results are encouraging, they are of limited value for generalization because of the small study population. Prospective randomized studies are required to evaluate the longterm efficacy of IGB treatment.

\section{Acknowledgment}

We express our grateful thanks to Mehmet Cemal Kahya, MD for statistical analysis.

\section{Disclosure}

The authors declared no conflicts of interest.

\section{References}

1 Nibben OG, Harboe H: Intragastric balloon as an artificial bezoar for treatment of obesity. Lancet 1982;i:198-199.

2 Genco A, Cipriano M, Bacci V, Cuzzolaro M, Materia A, Raparelli L, Docimo C, Lorenzo M: BioEnterics Intragastric Balloon (BIB): a shortterm, double-blind, randomised, controlled, crossover study on weight reduction in morbidly obese patients. Int J Obes 2006;30:129-133.

3 Kotzampassi K, Shrewsbury AD: Intragastric balloon: ethics, medical need and cosmetics. Dig Dis 2008;26:45-48.

4 Mathus-Vliegen EM: Intragastric balloon treatment for obesity: what does it really offer? Dig Dis 2008;26:40-44.

$\checkmark 5$ Imaz I, Martinez-Cervell C, Garcia-Alvarez EE, Sendra-Gutiérrez JM, González-Enríquez J: Safety and effectiveness of intragastric balloon for obesity. A meta-analysis. Obes Surg 2008;18:841-846.
6 Koutelidakis I, Dragoumis D, Papaziogas B, Patsas A, Katsougianopoulos A, Atmatzidis S, Atmatzidis $\mathrm{K}$ : Gastric perforation and death after the insertion of an intragastric balloon. Obes Surg 2009;19:393396

7 Mui WL, Ng EK, Tsung BY, Lam CH, Yung MY: Impact on obesity-related illnesses and quality of life following intragastric balloon. Obes Surg 2008; DOI 10.1007/s11695-008-9766-6.

8 Ohta M, Kitano S, Kai S, Shiromizu A, Eguchi H, Endo Y, Masaki T, Kakuma T, Yoshimatsu H: Initial Japanese experience with intragastric balloon placement. Obes Surg 2008;19:791-795.

9 Deitel M, Gawdat K, Melissas J: Reporting weight loss. Obes Surg 2007;17:565-568.

10 Göttig S, Daskalakis M, Weiner S, Weiner RA: Analysis of safety and efficacy of intragastric balloon in extremely obese patients. Obes Surg 2009; 19:677-683.

11 Pretolesi F, Redaelli G, Papagni L, Derchi LE: Intragastric balloon for morbid obesity causing chronic gastric dilatation. Eur Radiol 2001;11:588-589.
12 Cubattoli L, Barneschi C, Mastrocinque E, Bonucci P, Giomarelli PP: Cardiac arrest after intragastric balloon insertion in a super-obese patient. Obes Surg 2009;19:253-256.

13 Coskun H, Bostanci O, Dilege E, Bozbora A: BioEnterics intragastric balloon: clinical outcomes of the first 100 patients - a Turkish experience. Obes Surg 2008;18:1154-1156.

14 Herve J, Wahlen H, Schaeken A, Dallemagne B, Dewandre JM, Markiewicz S, Monami B, Weerts J, Jehaes O: What becomes of patients one year after the intragastric balloon has been removed? Obes Surg 2005;15:864-870.

15 Melissas J, Mouzas J, Filis D, Daskalakis M, Matrella E, Papadakis JA, Sevrisaianos N, Charalambides D: The intragastric balloon-smoothing the path to bariatric surgery. Obes Surg 2006;16:897-902.

16 Genco A, Cipriano M, Materia A, Bacci V, Maselli R, Musmeci L, Lorenzo M, Basso N: Laparoscopic sleeve gasrectomy versus intragastric balloon: a case-control study. Surg Endosc 2009;23:1849-1853. 